\title{
ON FUNCTIONALS OF A MARKED POISSON PROCESS OBSERVED BY A RENEWAL PROCESS
}

\author{
JEWGENI H. DSHALALOW and JEAN-BAPTISTE BACOT
}

(Received 22 May 2000)

\begin{abstract}
We study the functionals of a Poisson marked process $\Pi$ observed by a renewal process. A sequence of observations continues until $\Pi$ crosses some fixed level at one of the observation epochs (the first passage time). In various stochastic models applications (such as queueing with $N$-policy combined with multiple vacations), it is necessary to operate with the value of $\Pi$ prior to the first passage time, or prior to the first passage time plus some random time. We obtain a time-dependent solution to this problem in a closed form, in terms of its Laplace transform. Many results are directly applicable to the time-dependent analysis of queues and other stochastic models via semi-regenerative techniques.
\end{abstract}

2000 Mathematics Subject Classification. 60K10, 60K15, 60K25.

1. Introduction. This paper investigates a class of functionals of a Poisson marked process $\Pi$ observed by a renewal process $\tau$ (and thereby forming a marked renewal process). It is of interest to see the process $\Pi$ cross some fixed threshold which, however, may be observed only later by $\tau$. The value of $\Pi$ at the first passage time, as observed by $\tau$, is known as the first excess level. We are targeting joint functionals of the Poisson process $\Pi$, prior to the first passage time, and of the first excess level as well as the value of $\Pi$, between the first passage time and some other instant generated by a given random variable.

Situations like these frequently occur in stochastic models, particularly in queuing. The renewal process observing the Poisson process can represent multiple vacations rendered by a server $[2,3,12,13,14,15,16,17,19]$. In other words, when the server leaves the system, it generates a sequence of vacation (or maintenance) segments each of which ends up with the server returning to the system and checking on its status. The whole vacation period is suspended (until its next occurrence, when the queue is exhausted or, in a quorum option [4, 9, 10, 13], drops below some specific level) whenever the system accumulates to some fixed number of units or more and this prompts the server to resume his work $[1,4,9,10,11,18]$. When using semiregenerative analysis it is imperative to know the status of the system on the period prior to the completion of the first service. This period is referred to as the first service cycle and it consists of the whole vacation period and service period, separated by the first passage time. Evaluation of such a functional is generally a lion portion of the semi-regenerative analysis of many stochastic models and a unified treatment of this subject would be advantageous. 
Studies on crossing level analysis were earlier rendered in Dshalalow [5, 6, 7, 8], but they were not applied to time-dependent processes. In the present paper, when examining such functionals, we arrive at compact analytic formulas of time-dependent discrete-valued processes, in terms of Laplace transforms (promising to yield closed forms also for their continuous-valued compounds). Many results are directly applicable to time-dependent analysis of semi-regenerative processes occurring in queues and other stochastic models.

2. First passage time of a basic delayed marked renewal process. In this section, for consistency, we give some preliminaries on a class of marked delayed renewal processes pertinent to the upcoming sections. The below results are a modification of those in Dshalalow $[6,7,8]$ and therefore somewhat new.

All stochastic processes are considered on a probability space $(\Omega, \mathscr{F}, \mathbb{P})$. Let

$$
(\mathbb{A}, \tau)=\sum_{n=0}^{\infty} X_{n} \varepsilon_{\tau_{n}}
$$

be a delayed marked renewal process with position dependent marking where $\varepsilon_{a}$ is a point mass. We assume that the marks $X_{0}, X_{1}, \ldots$ are nonnegative integer-valued. The point process $\tau=\left\{\tau_{0}, \tau_{1}, \ldots\right\}$ is placed on the real axis and it is related to the following random variables and functionals.

The sequence $\left\{\chi_{n}=\tau_{n}-\tau_{n-1}, X_{n} ; n=1, \ldots\right\}$ is a sequence of i.i.d. two-variate random variables with the joint common transformation

$$
\gamma(z, \theta)=\mathbb{E}\left[z^{X_{1}} e^{-\theta X_{1}}\right], \quad|z|<1, \operatorname{Re}(\theta) \geq 0,
$$

marginal probability distribution function (PDF) $V=V(t), t \geq 0$, of $\chi_{1}$, and its LaplaceStieltjes transform (LST)

$$
\gamma(\theta)=\gamma(1, \theta)
$$

Let

$$
\mu(z, \theta)=\mathbb{E}\left[z^{X_{0}} e^{-\theta \chi_{0}}\right], \quad|z|<1, \operatorname{Re}(\theta) \geq 0,
$$

with marginal PDF $V_{0}=V_{0}(t), t \geq 0$, of $\chi_{0}$, its LST

$$
\mu(\theta)=\mu(1, \theta)
$$

and probability generating function (pgf) of $X_{0}$,

$$
m(z)=\mu(z, 0) .
$$

With the notation $A_{k}=X_{0}+\cdots+X_{k}$, we have $\left\{A_{k}\right\}$ as the marginal delayed (discretevalued) renewal process of $(\mathbb{A}, \tau)$.

Now, let $N$ be a fixed positive integer, which $(\mathbb{A}, \tau)$ is supposed to cross at one of the epochs $\tau_{0}, \tau_{1}, \ldots$, called the first passage time. More formally, let $v=\inf \{k=0,1, \ldots$ : $\left.A_{k} \geq N\right\}$ be known, [6], as the termination index. Then $\tau_{v}$ is the first passage time and $A_{v}$ is the first excess level. We are interested in the functional

$$
L(\xi, x, z, \theta)=L(\xi, x, z, \theta ; N)=\mathbb{E}\left[\xi^{v} x^{A_{\nu-1}} z^{A_{v}} e^{-\theta \tau_{v}}\right],
$$


where $N$ is the above control threshold of the termination index, pre-first excess level, first excess level, and the first passage time, respectively. The value of the pre-first excess level may be of independent interest, as it informs us one step ahead of some "catastrophe."

In what follows, we will need the following transformation:

$$
D_{p} f(w)=(1-w) \sum_{p \geq 0} w^{p} f(p), \quad|w|<1,
$$

where $f$ is an integrable Borel measurable function. (Notice that while $p$ is a dummy variable, it is being used in the notation $D_{p}$ for convenience.)

With Taylor-like functional

$$
\mathscr{D}_{w}^{k}(\cdot)= \begin{cases}\lim _{w \rightarrow 0} \frac{1}{k !} \frac{\partial^{k}}{\partial w^{k}} \frac{1}{1-w}(\cdot), & k \geq 0, \\ 0, & k<0,\end{cases}
$$

we can restore $f$ subject to $D_{p}$

$$
\mathscr{D}_{w}^{k}\left(D_{p} f(w)\right)=f(k) .
$$

The subscript $w$ in $\mathscr{D}$ serves also the same purpose as $p$. For various special cases throughout the remainder of this paper, we notice a few elementary properties of the operator $\mathscr{D}_{y}^{p}$ (see Dshalalow [8])

$\mathscr{D}_{y}^{p}$ is a linear operator with fixed points at every constant function.

For any function $h$, analytic at zero,

$$
\mathscr{D}_{y}^{p}\left(y^{k} h(y)\right)= \begin{cases}0, & p<k, \\ \mathscr{D}_{y}^{p-k}(h(y)), & p \geq k .\end{cases}
$$

Now, given the process $(\mathbb{A}, \tau)$, we introduce the auxiliary sequence of random variables

$$
\left\{v_{p}=\inf \left\{k: A_{k}>p\right\}: p=0,1, \ldots\right\} \quad\left(\text { of which } v_{N-1}=v\right),
$$

and apply the transformation $D_{p}$ to the sequence $\{L(\xi, x, z, \theta ; p+1)\}_{p}$. Then, $L(\xi, x, z, \theta ; N)$ can be traced back by means of the operator $\mathscr{D}_{w}^{N-1}$. All this is subject to the following theorem.

THEOREM 2.1. The functional $L$ satisfies the following formula:

$$
L(\xi, x, z, \theta ; N)=\mu(z, \theta)-\mathscr{D}_{w}^{N-1}\left\{\mu(w z, \theta)-\xi \mu(w x z, \theta) \frac{\gamma(z, \theta)-\gamma(w z, \theta)}{1-\xi \gamma(w x z, \theta)}\right\},
$$

where $L, \mu, \gamma$, and $\mathscr{D}$ are defined in (2.2), (2.4), (2.7), and (2.9), respectively.

Proof. First, observe that

$$
D_{p}\left(\mathbf{1}_{\left\{v_{p}=j\right\}}\right)(w)=w^{A_{j-1}}-w^{A_{j}}, \quad j=0,1, \ldots
$$


(which is due to $[6,8]$ or it can also be easily verified), where $A_{-1}=0$ and $\mathbf{1}_{A}$ is the indicator function of a set $A$. Then, from (2.15),

$$
\begin{aligned}
D_{p}\{L(\xi, x, z, \theta ; p+1)\}(w) & =\Lambda(\xi, x w, z, \theta)-\Lambda(\xi, x, z w, \theta) \\
& =\sum_{n=0}^{\infty} \xi^{n} \Lambda_{n}(x w, z, \theta)-\sum_{n=0}^{\infty} \xi^{n} \Lambda_{n}(x, w z, \theta),
\end{aligned}
$$

where

$$
\Lambda_{n}(x, z, \theta)=\mathbb{E}\left[x^{A_{n-1}} z^{A_{n}} e^{-\theta \tau_{n}}\right] .
$$

Clearly,

$$
\Lambda_{0}(x, z, \theta)=\mathbb{E}\left[z^{A_{0}} e^{-\theta \tau_{0}}\right]=\mu(z, \theta),
$$

while

$$
\Lambda_{n}(x, z, \theta)=\mathbb{E}\left[(x z)^{A_{n-1}} z^{X_{n}} e^{-\theta \tau_{n-1}} e^{-\theta x_{n}}\right] .
$$

The latter easily reduces to

$$
\mu(x z, \theta) \gamma^{n-1}(x z, \theta) \gamma(z, \theta)
$$

which leads to

$$
\Lambda(\xi, x, z, \theta)=\mu(z, \theta)+\mu(x z, \theta) \gamma(z, \theta) \xi \frac{1}{1-\xi \gamma(x z, \theta)},
$$

and then to

$$
D_{p}\{L(\xi, x, z, \theta ; p+1)\}(w)=\mu(z, \theta)-\mu(w z, \theta)+\xi \mu(w x z, \theta) \frac{\gamma(z, \theta)-\gamma(w z, \theta)}{1-\xi \gamma(w x z, \theta)} .
$$

The rest is due to (2.11).

Of course, the parameter $N$ in the functional $L(\xi, x, z, \theta ; N)$ can be dropped throughout the rest of the paper, as it was used only temporarily.

We make use of the following special cases.

CASE 1. Omitting the pre-first excess level, that is, setting $x=1$, simplifies (2.14)

$$
L(\xi, 1, z, \theta)=\mu(z, \theta)-[1-\xi \gamma(z, \theta)] \mathscr{D}_{w}^{N-1}\left\{\frac{\mu(w z, \theta)}{1-\xi \gamma(w z, \theta)}\right\} .
$$

CASE 2. Let

$$
\Pi=\sum_{j=1}^{\infty} U_{j} \varepsilon_{r_{j}}
$$

be a stationary compound Poisson process of intensity $\lambda$ and with $a(z)$ as the common pgf of marks $U_{1}, U_{2}, \ldots$ (arriving batches) and let

$$
\Pi_{0}=X_{0} \varepsilon_{0}+\Pi
$$

be "observed" by $\tau$ at epochs $\tau_{0}, \tau_{1}, \ldots$, so that $\tau_{0}=0$. Then, with

$$
A_{0}=X_{0}, \quad A_{n}=\Pi_{0}\left(\left[0, \tau_{n}\right]\right), \quad X_{n}=\Pi_{0}\left(\left(\tau_{n-1}, \tau_{n}\right]\right), \quad n=1,2, \ldots,
$$


we have

$$
(\mathbb{A}, \tau)=\sum_{n=0}^{\infty} X_{n} \varepsilon_{\tau_{n}}
$$

as the delayed marked renewal process embedded in $\Pi_{0}$. Abbreviating

$$
\lambda(z, \theta)=\lambda-\lambda a(z)+\theta
$$

we have by standard probability arguments that $\gamma(z, \theta)$ defined in (2.2) and (2.3) is

$$
\gamma(z, \theta)=\gamma(\lambda(z, \theta)) .
$$

Then, from Theorem 2.1, we have the following corollary.

COROLLARY 2.2. Under the assumptions (2.24), (2.25), (2.26), and (2.27), the functional $L$ (defined in (2.7)) reduces to

$$
L(\xi, x, z, \theta)=m(z)-\mathscr{D}_{w}^{N-1}\left\{m(w z)-\xi m(w x z) \frac{\gamma(\lambda(z, \theta))-\gamma(\lambda(w z, \theta))}{1-\xi \gamma(\lambda(w x z, \theta))}\right\},
$$

where $m, \gamma$, and $\mathscr{D}$ are subject to (2.6), (2.9), (2.28), and (2.29), respectively. In particular, with $X_{0}=i$ a.s. or $m(z)=z^{i}$ and properties (2.12), (2.30) reduce to

$$
L(\xi, x, z, \theta)=\xi(x y z)^{i} \mathscr{D}_{w}^{N-i-1}\left\{\frac{\gamma(\lambda(y z, \theta))-\gamma(\lambda(w y z, \theta))}{1-\xi \gamma(\lambda(w x y z, \theta))}\right\} .
$$

On the other hand, setting $x=1$ in (2.30) (also, in agreement with the special case of (2.23) for $\tau_{0}=0$ ) yields

$$
L(\xi, 1, z, \theta)=m(z)-[1-\xi \gamma(\lambda(z, \theta))] \mathscr{D}_{w}^{N-1}\left\{\frac{m(w z)}{1-\xi \gamma(\lambda(w z, \theta))}\right\} .
$$

A further special case of (2.32) (frequently encountered in applications) is when $X_{0}=i$ a.s. By (2.12),

$$
L(\xi, 1, z, \theta)=z^{i}-z^{i}[1-\xi \gamma(\lambda(z, \theta))] \mathscr{D}_{w}^{N-i-1}\left\{\frac{1}{1-\xi \gamma(\lambda(w z, \theta))}\right\} .
$$

Notice that formulas (2.32) and (2.33) (that are previously known from Dshalalow [6]) can be applied to queuing models of type $M^{X} / G / 1$ with server vacations and $N$-policy. Formula (2.33) will then represent the functional of the queuing process (initiated at level $i$ ) at the end of a vacation period and the first passage time on a vacation period $\left[0, \tau_{v}\right]$ generated by multiple vacations.

3. Poisson process observed by a renewal process. Throughout the rest of this paper, we continue examining the fluctuations of $\Pi_{0}$ at the observation epochs $\tau$ about level $N$. In some applications, we need to know the value of the Poisson process at some moment of time $t$ prior to the first passage time and at some moment between the first passage time and the end of a period of time following the first passage time. It 
frequently takes place when studying various classes of semi-regenerative processes, such as those in queuing. Under the assumptions of Case 2, we target the functional

$$
H(\xi, x, y, z, \theta)=\int_{0}^{\infty} e^{-\theta t} \mathbb{E}\left[\xi^{v} x^{A_{v-1}} y^{A_{v}} z^{N_{t}} \mathbf{1}_{\left\{\tau_{v} \leq t\right\}} \mathbf{1}_{\left\{\tau_{v}+\Sigma>t\right\}}\right] d t
$$

where $\mathbf{1}_{A}$ is the indicator function of a set $A, \Sigma$ is a nonnegative random variable, independent of $\tau$ and $\Pi_{0}$, with the PDF $S$ and LST $\sigma$, and $N_{t}=\Pi_{0}([0, \mathrm{t}])$. Note that if $\Sigma=\infty$, then $\mathbf{1}_{\left\{\tau_{v}+\Sigma>t\right\}}$ degenerates to 1 and $S=\sigma=\mathbf{0}$.

First we prove the following lemma.

LEMMA 3.1. The functional $h$ defined as

$$
h(\xi, x, y, z, \theta)=\sum_{n=0}^{\infty} \int_{0}^{\infty} e^{-\theta t} \mathbb{E}\left[\xi^{n} x^{A_{n-1}} y^{A_{n}} z^{N_{t}} \mathbf{1}_{\left\{\tau_{n} \leq t\right\}} \mathbf{1}_{\left\{\tau_{n}+\Sigma>t\right\}}\right] d t
$$

(with $A_{-1}:=0$ ) satisfies the following formula:

$$
\begin{array}{r}
h(\xi, x, y, z, \theta)=\frac{1-\sigma(\lambda(z, \theta))}{\lambda(z, \theta)}\left[m(y z)+m(x y z) \frac{\xi \gamma(\lambda(y z, \theta))}{1-\xi \gamma(\lambda(x y z, \theta))}\right], \\
\|z\|<1,\|x y\| \leq 1, \operatorname{Re}(\theta) \geq 0,
\end{array}
$$

where $m(z), \lambda(z, \theta)$, and $\gamma(\theta)$ are due to (2.6), (2.28), and (2.29), respectively.

PROOF. (1) For $n>0$,

$$
\begin{aligned}
& h_{n}(x, y, z, \theta)=\int_{0}^{\infty} e^{-\theta t} \mathbb{E}\left[\mathbf{1}_{\left\{\tau_{n} \leq t\right\}} \mathbf{1}_{\left\{\tau_{n}+\Sigma>t\right\}} \mathbb{E}\left[x^{N_{\tau_{n-1}}} y^{N_{\tau_{n}}} z^{N_{t}} \mid \tau_{n-1}, \tau_{n}, \Sigma\right]\right] d t \\
& =\int_{0}^{\infty} e^{-\theta t} \mathbb{E}\left[\mathbf{1}_{\left\{\tau_{n} \leq t\right\}} \mathbf{1}_{\left\{\tau_{n}+\Sigma>t\right\}} \mathbb{E}\left[x^{N_{\tau_{n-1}}} y^{N_{\tau_{n}}} z^{N_{t}} \mid \tau_{n-1}, \tau_{n}\right]\right] d t \\
& =m(x y z) \int_{0}^{\infty} e^{-\theta t} \mathbb{E}\left[\mathbf{1}_{\left\{\tau_{n} \leq t\right\}} \mathbf{1}_{\left\{\tau_{n}+\Sigma>t\right\}} e^{\lambda \tau_{n-1}[a(x y z)-1]}\right. \\
& \left.\times e^{\lambda \chi_{n}[a(y z)-1]} e^{\lambda\left(t-\tau_{n-1}-\chi_{n}\right)[a(z)-1]}\right] d t \\
& =m(x y z) \int_{t=0}^{\infty} \int_{u=0}^{t} \int_{s=0}^{t-u} \int_{\xi=t-u-s}^{\infty} e^{-\theta t} e^{\lambda u[a(x y z)-1]} e^{\lambda s[a(y z)-1]} \\
& \times e^{\lambda(t-u-s)[a(z)-1]} S(d \xi) V(d s) V^{(n-1) *}(d u) d t \\
& =m(x y z) \int_{u=0}^{\infty} e^{\lambda u[a(x y z)-1]-\theta u} \int_{s=0}^{\infty} e^{\lambda s[a(y z)-1]-\theta s} \\
& \times \int_{\xi=0}^{\infty} \int_{c=0}^{\xi} e^{\lambda c[a(z)-1]-\theta c} d c S(d \xi) V(d s) V^{(n-1) *}(d u) \\
& =m(x y z) \frac{1-\sigma(\lambda(z, \theta))}{\lambda(z, \theta)} \gamma(\lambda(y z, \theta)) \gamma^{n-1}(\lambda(x y z), \theta) \text {. }
\end{aligned}
$$

Note that $z \mapsto e^{\lambda(z, \theta)(s-c)}$ is an $L^{1}$-function for all $\|z\|<1$ and $\operatorname{Re}(\theta) \geq 0$ (as, by Schwarz lemma, it makes $\|a(z)\|<1$ ) and hence its integral on $[s, \infty)$ is convergent for $\|z\|<1$ and $\operatorname{Re}(\theta) \geq 0$.

(2) For $n=0$, we easily verify that

$$
h_{0}(x, y, z, \theta)=\int_{0}^{\infty} e^{-\theta t} \mathbb{E}\left[\mathbf{1}_{\{\Sigma>t\}} y^{N_{0}} z^{N_{t}}\right] d t=m(y z) \frac{1-\sigma(\lambda(z, \theta))}{\lambda(z, \theta)} .
$$


Finally, summing up all $h_{n}(x, y, z, \theta)$ 's we have (3.3).

THEOREM 3.2. The functional

$$
H(\xi, x, y, z, \theta)=\int_{0}^{\infty} e^{-\theta t} \mathbb{E}\left[\xi^{v} x^{A_{\nu-1}} y^{A_{v}} z^{N_{t}} \mathbf{1}_{\left\{\tau_{\nu} \leq t\right\}} \mathbf{1}_{\left\{\tau_{v}+\Sigma>t\right\}}\right] d t
$$

satisfies the following formula:

$$
H(\xi, x, y, z, \theta)=\frac{1-\sigma(\lambda(z, \theta))}{\lambda(z, \theta)} L(\xi, x, y z, \theta),
$$

where $L(\xi, x, z, \theta)=\mathbb{E}\left[\xi^{v} x^{A_{v-1}} z^{A_{v}} e^{-\theta \tau_{v}}\right]$ satisfies (2.30).

Proof. As in the case of $L$, we temporarily adhere $N$ in notation of $H(\xi, x, y, z, \theta ; N)$. Obviously,

$$
H(\xi, x, y, z, \theta ; N)=\sum_{n=0}^{\infty} \int_{0}^{\infty} e^{-\theta t} \mathbb{E}\left[\xi^{v} \mathbf{1}_{\{v=n\}} x^{A_{n-1}} y^{A_{n}} z^{N_{t}} \mathbf{1}_{\left\{\tau_{n} \leq t\right\}} \mathbf{1}_{\left\{\tau_{v}+\Sigma>t\right\}}\right] d t,
$$

with $A_{-1}:=0$. Now, we apply the transformation $D_{p}$ to the functional $H(\xi, x, y, z$, $\theta ; p+1)$, in notation $D_{p} H(\xi, x, y, z, \theta ; p+1)(w)$ by replacing, as in (2.13), $v$ by the auxiliary sequence $\left\{v_{p}=\inf \left\{k: A_{k}>p\right\}: p=0,1, \ldots\right\}$ of random variables in each of the functionals $H(\xi, x, y, z, \theta ; p+1)$ 's. Because of (2.15),

$$
\begin{aligned}
& D_{p} H(\xi, x, y, z, \theta ; p+1)(w) \\
& =\sum_{n=0}^{\infty} \xi^{n} \int_{0}^{\infty} e^{-\theta t}\left\{\mathbb{E}\left[(w x)^{A_{n-1}} y^{A_{n}} z^{N_{t}} \mathbf{1}_{\left\{\tau_{n} \leq t\right\}} \mathbf{1}_{\left\{\tau_{n}+\Sigma>t\right\}}\right]\right. \\
& \left.-\mathbb{E}\left[x^{A_{n-1}}(w y)^{A_{n}} z^{N_{t}} \mathbf{1}_{\left\{\tau_{n} \leq t\right\}} \mathbf{1}_{\left\{\tau_{n}+\Sigma>t\right\}}\right]\right\} d t,
\end{aligned}
$$

which yields

$$
D_{p} H(\xi, x, y, z, \theta ; p+1)(w)=h(\xi, w x, y, z, \theta)-h(\xi, x, w y, z, \theta),
$$

with $h$ defined in (3.2) of Lemma 3.1 and hence, by (3.3),

$$
\begin{aligned}
& D_{p} H(\xi, x, y, z, \theta ; p+1)(w) \\
& =\frac{1-\sigma(\lambda(z, \theta))}{\lambda(z, \theta)}\left[m(y z)-m(w y z)+\xi m(w x y z) \frac{\gamma(\lambda(y z, \theta))-\gamma(\lambda(w y z, \theta))}{1-\xi \gamma(\lambda(w x y z, \theta))}\right] .
\end{aligned}
$$

Finally, we restore $H(\xi, x, y, z, \theta ; N)$ by using the operator $\mathscr{D}_{w}^{k}$ for $k=N-1$ and its property (2.11).

From (2.30), we have the following corollary.

COROLLARY 3.3. If $m(z)=z^{i}$, then formula (3.7) reduces to

$$
H(\xi, x, y, z, \theta)=\frac{1-\sigma(\lambda(z, \theta))}{\lambda(z, \theta)} L(\xi, x, y z, \theta),
$$

with the version of $L$ in the form

$$
L(\xi, x, y z, \theta)=\xi(x y z)^{i} \mathscr{D}_{w}^{N-i-1}\left\{\frac{\gamma(\lambda(y z, \theta))-\gamma(\lambda(w y z, \theta))}{1-\xi \gamma(\lambda(w x y z, \theta))}\right\} .
$$


In addition, for $x=1$,

$$
\begin{aligned}
& H(\xi, 1, y, z, \theta) \\
& =\int_{0}^{\infty} e^{-\theta t} \mathbb{E}\left[\xi^{v} y^{A_{v}} z^{N_{t}} \mathbf{1}_{\left\{\tau_{v} \leq t\right\}} \mathbf{1}_{\left\{\tau_{v}+\Sigma>t\right\}}\right] d t \\
& =\frac{[1-\sigma(\lambda(z, \theta))]\left\{(y z)^{i}-(y z)^{i}[1-\xi \gamma(\lambda(y z, \theta))]\right\}}{\lambda(z, \theta)} \mathscr{D}_{w}^{N-i-1}\left\{\frac{1}{1-\xi \gamma(\lambda(w x y z, \theta))}\right\},
\end{aligned}
$$

in which we identify

$$
L(\xi, 1, z, \theta)=z^{i}-z^{i}[1-\xi \gamma(\lambda(z, \theta))] \mathscr{D}_{w}^{N-i-1}\left\{\frac{1}{1-\xi \gamma(\lambda(w z, \theta))}\right\} .
$$

COROLLARY 3.4. For $\Sigma=\infty$ a.s., as we previously noticed, $S, \sigma$, and $b$ vanish. Thus, from Theorem 3.2,

$$
G(\xi, x, y, z, \theta)=\int_{0}^{\infty} e^{-\theta t} \mathbb{E}\left[\xi^{v} x^{A_{v-1}} y^{A_{v}} z^{N_{t}} \mathbf{1}_{\left\{\tau_{v} \leq t\right\}}\right] d t=\frac{1}{\lambda(z, \theta)} L(\xi, x, y z, \theta),
$$

where L satisfies (2.30).

COROLLARY 3.5. Taking $\xi=x=y=1$ in (2.30) and (3.16),

$$
G(1,1,1, z, \theta)=\int_{0}^{\infty} e^{-\theta t} \mathbb{E}\left[z^{N_{t}} \mathbf{1}_{\left\{\tau_{\nu} \leq t\right\}}\right] d t=\frac{1}{\lambda(z, \theta)} L(1,1, z, \theta),
$$

or in the form

$$
G(1,1,1, z, \theta)=\frac{1}{\lambda(z, \theta)}\left[m(z)-[1-\gamma(\lambda(z, \theta))] \mathscr{D}_{w}^{N-1}\left\{\frac{m(w z)}{1-v(w z, \theta)}\right\}\right] .
$$

Then $F(z, \theta)=\int_{0}^{\infty} e^{-\theta t} \mathbb{E}\left[z^{N_{t}} \mathbf{1}_{\left\{\tau_{v}>t\right\}}\right] d t$ as

$$
\begin{aligned}
F(z, \theta) & =\int_{0}^{\infty} e^{-\theta t} \mathbb{E}\left[z^{N_{t}}\right] d t-G(1,1,1, z, \theta) \\
& =\frac{1}{\lambda(z, \theta)}[1-\gamma(\lambda(z, \theta))] D_{w}^{N-1}\left\{\frac{m(w z)}{1-\gamma(\lambda(w z, \theta))}\right\},
\end{aligned}
$$

or in the form

$$
F(z, \theta)=\frac{1}{\lambda(z, \theta)}[m(z)-L(1,1, z, \theta)] .
$$

In particular, for $m(z)=z^{i}$,

$$
F(z, \theta)=\frac{z^{i}}{\lambda(z, \theta)}[1-\gamma(\lambda(z, \theta))] \mathscr{D}_{w}^{N-i-1}\left\{\frac{1}{1-\gamma(\lambda(w z, \theta))}\right\},
$$

or in the form

$$
F(z, \theta)=\frac{1}{\lambda(z, \theta)}\left[z^{i}-L(1,1, z, \theta)\right]
$$


COROLLARY 3.6. The functional

$$
\Gamma(z, \theta)=\int_{0}^{\infty} e^{-\theta t} \mathbb{E}\left[z^{N_{t}} \mathbf{1}_{\left\{\tau_{v}+\Sigma>t\right\}}\right] d t
$$

equals

$$
\begin{aligned}
\Gamma(z, \theta) & =F(z, \theta)+H(1,1,1, z, \theta) \\
& =\int_{0}^{\infty} e^{-\theta t} \mathbb{E}\left[z^{N_{t}} \mathbf{1}_{\left\{\tau_{v}>t\right\}}\right] d t+\int_{0}^{\infty} e^{-\theta t} \mathbb{E}\left[z^{N_{t}} \mathbf{1}_{\left\{\tau_{v} \leq t\right\}} \mathbf{1}_{\left\{\tau_{\nu}+\Sigma>t\right\}}\right] d t \\
& =\frac{1}{\lambda(z, \theta)}[m(z)-L(1,1 z, \theta)]+\frac{1-\sigma(\lambda(z, \theta))}{\lambda(z, \theta)} L(1,1, z, \theta) \\
& =\frac{m(z)-\sigma(\lambda(z, \theta)) L(1,1, z, \theta)}{\lambda(z, \theta)},
\end{aligned}
$$

or, when $m(z)=z^{i}$,

$$
\Gamma(z, \theta)=\frac{z^{i}-\sigma(\lambda(z, \theta)) L(1,1, z, \theta)}{\lambda(z, \theta)} .
$$

REMARK 3.7. Formula (3.25) takes place in queuing when, by using semiregenerative techniques, one needs to evaluate the functional of the queue length on the first service cycle $\left[0, \tau_{v}+\Sigma_{1}\right.$ ), which consists of $\left[0, \tau_{v}\right]$ (and can be regarded as a "multiple vacation period") and of the period $\left(\boldsymbol{\tau}_{v}, \boldsymbol{\tau}_{v}+\Sigma_{1}\right)$, where $\Sigma_{1}$ is the duration of the first service. For this particular application, we need to set $\theta=0$ and thereby have $L(1,1, z, \theta)$ reduced to the marginal functional of the first excess level.

ACKNOWLEDGEMENTs. The authors are very grateful to Lajos Takács for his very valuable comments and many helpful discussions.

\section{REFERENCES}

[1] K. C. Chae and H. W. Lee, $M^{X} / G / 1$ vacation models with $N$-policy: heuristic interpretation of the mean waiting time, J. Oper. Res. Soc. 46 (1995), no. 2, 258-264. Zbl 832.60090.

[2] B. Doshi, Single server queues with vacations, Stochastic Analysis of Computer and Communication Systems, North-Holland, Amsterdam, 1990, pp. 217-265. CMP 1150292.

[3] B. T. Doshi, Queueing systems with vacations - a survey, Queueing Systems Theory Appl. 1 (1986), no. 1, 29-66. MR 89b:60212. Zbl 655.60089.

[4] J. H. Dshalalow, On a first passage problem in general queueing systems with multiple vacations, J. Appl. Math. Stochastic Anal. 5 (1992), no. 2, 177-192. MR 93k:60225. Zbl 755.60080.

[5] _ On termination time processes, Studies in Applied Probability (J. Galambos and J. Gani, eds.), vol. 31A, Applied Probability Trust, Sheffield, 1994, Essays in honor of Lajos Takács, J. Appl. Probab., pp. 325-336. Zbl 805.60092.

[6] _ Excess level processes in queueing, Advances in Queueing (J. H. Dshalalow, ed.), Probability and Stochastics Series, CRC press, Florida, 1995, pp. 243-262. MR 97d:60145. Zbl 881.60078.

[7] _ On the level crossing of multidimensional delayed renewal processes, J. Appl. Math. Stochastic Anal. 10 (1997), no. 4, 355-361. CMP 1488 018. Zbl 896.60056. 
[8]__ Queueing systems with state dependent parameters, Frontiers in Queueing (J. H. Dshalalow, ed.), Probability and Stochastics Series, CRC press, Florida, 1997, pp. 61-116. MR 97k:60244. Zbl 871.60076.

[9] _ Queues with hysteretic control by vacation and post-vacation periods, Queueing Systems Theory Appl. 29 (1998), no. 2-4, 231-268. MR 2000d:60147. Zbl 915.90111.

[10] J. H. Dshalalow and J. Yellen, Bulk input queues with quorum and multiple vacations, Math. Probl. Eng. 2 (1996), no. 2, 95-106. Zbl 921.60080.

[11] A. Federgruen and K. C. So, Optimality of threshold policies in single-server queueing systems with server vacations, Adv. in Appl. Probab. 23 (1991), no. 2, 388-405. MR 92h:90056. Zbl 728.60088.

[12] C. M. Harris and W. G. Marchal, State dependence in $M / G / 1$ server-vacation models, Oper. Res. 36 (1988), no. 4, 560-565. MR 89g:60280. Zbl 652.90046.

[13] M. J. Jacob and T. P. Madhusoodanan, Transient solution for a finite capacity $M / G^{a, b} / 1$ queueing system with vacations to the server, Queueing Systems Theory Appl. 2 (1987), no. 4, 381-386. MR 89k:60138. Zbl 654.60087.

[14] J. Keilson and L. D. Servi, Dynamics of the $M / G / 1$ vacation model, Oper. Res. 35 (1987), no. 4, 575-582. MR 89g:60285. Zbl 636.90033.

[15] O. Kella, Optimal control of the vacation scheme in an $M / G / 1$ queue, Oper. Res. 38 (1990), no. 4, 724-728. CMP 1067 472. Zbl 719.90033.

[16] H.-S. Lee, Optimal control of the $M^{X} / G / 1 / K$ queue with multiple server vacations, Comput. Oper. Res. 22 (1995), no. 5, 543-552. Zbl 838.90047.

[17] H. W. Lee, S. S. Lee, and K. C. Chae, A fixed-size batch service queue with vacations, J. Appl. Math. Stochastic Anal. 9 (1996), no. 2, 205-219. MR 96m:60223. Zbl 858.60085.

[18] H. W. Lee, S. S. Lee, J. O. Park, and K. C. Chae, Analysis of the $M^{X} / G / 1$ queue with $N$-policy and multiple vacations, J. Appl. Probab. 31 (1994), no. 2, 476-496. MR 95a:60125. Zbl 804.60081.

[19] H. Takagi, Time-dependent process of $M / G / 1$ vacation models with exhaustive service, J. Appl. Probab. 29 (1992), no. 2, 418-429. MR 93c:60153. Zbl 753.60096.

JeWgeni H. DShalalow: ApPlied MATHematics Program, Florida INSTITUTE OF TeCHNOLOGY, MELBOURNE, FL 32901, USA

E-mail address: eugene_d@be11 south.net

Jean-Baptiste Bacot: Operations Research Program, Florida Institute of TeChNOLOGY, MELBOURNE, FL 3201, USA 


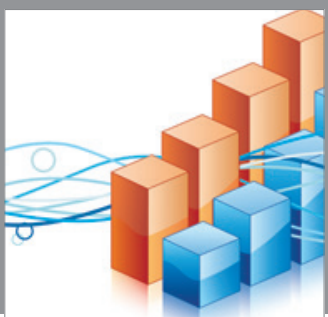

Advances in

Operations Research

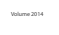

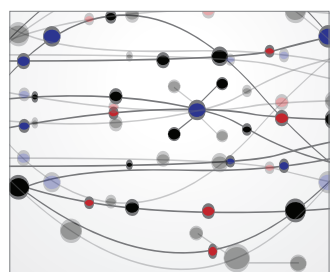

\section{The Scientific} World Journal
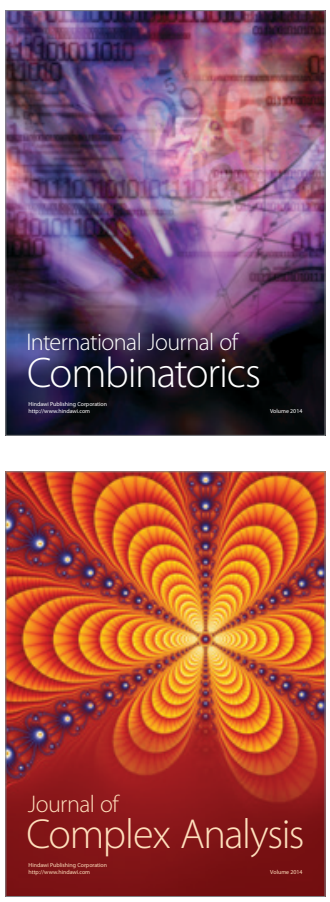

International Journal of

Mathematics and

Mathematical

Sciences
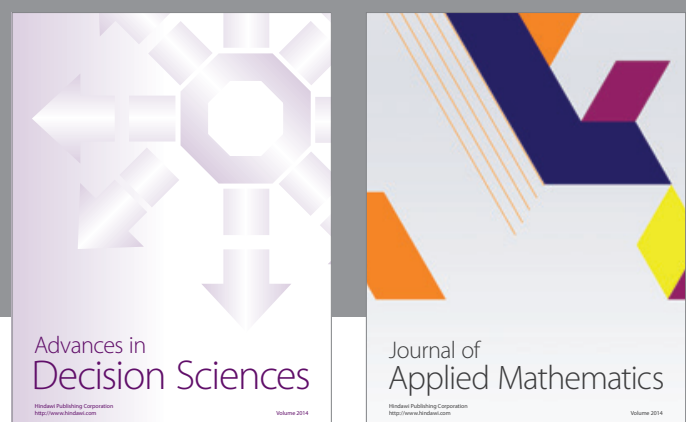

Journal of

Applied Mathematics
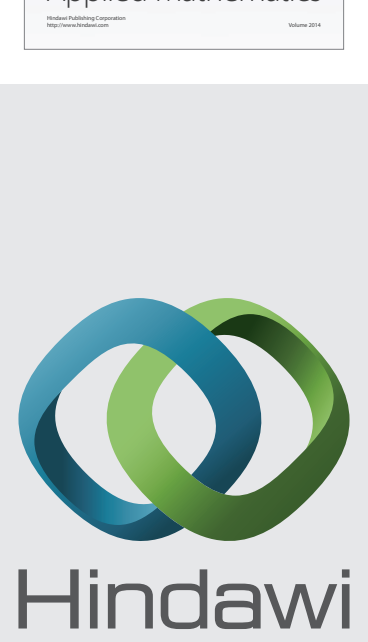

Submit your manuscripts at http://www.hindawi.com
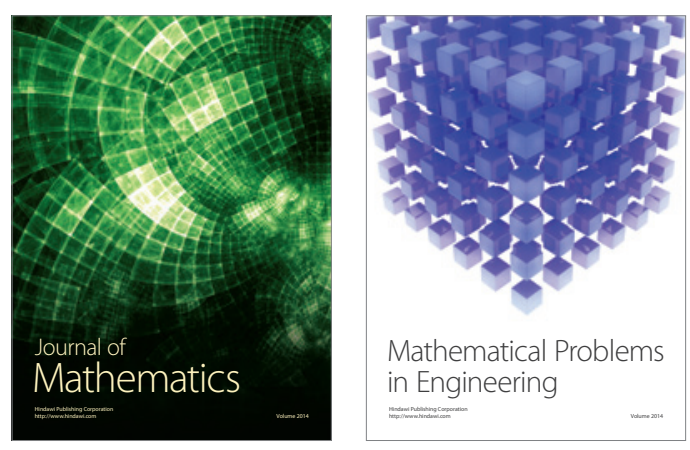

Mathematical Problems in Engineering
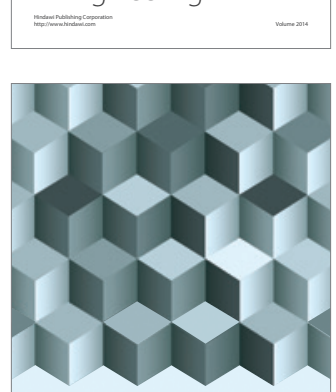

Journal of

Function Spaces
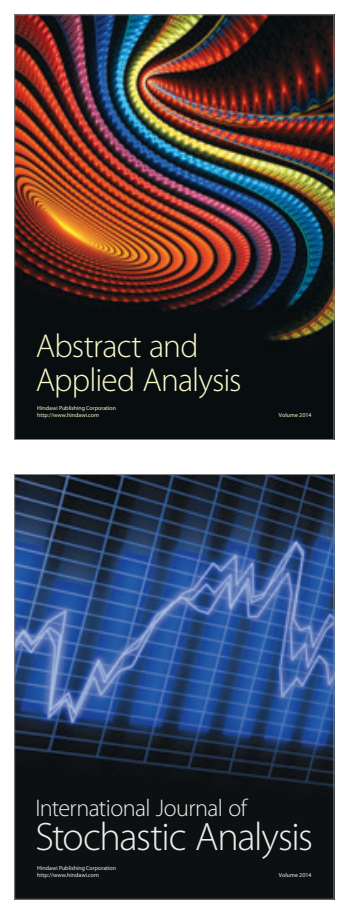

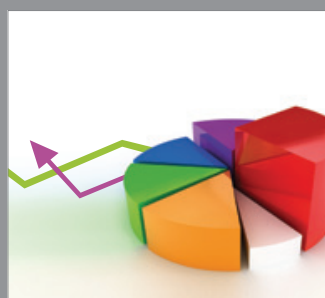

ournal of

Probability and Statistics

Promensencen
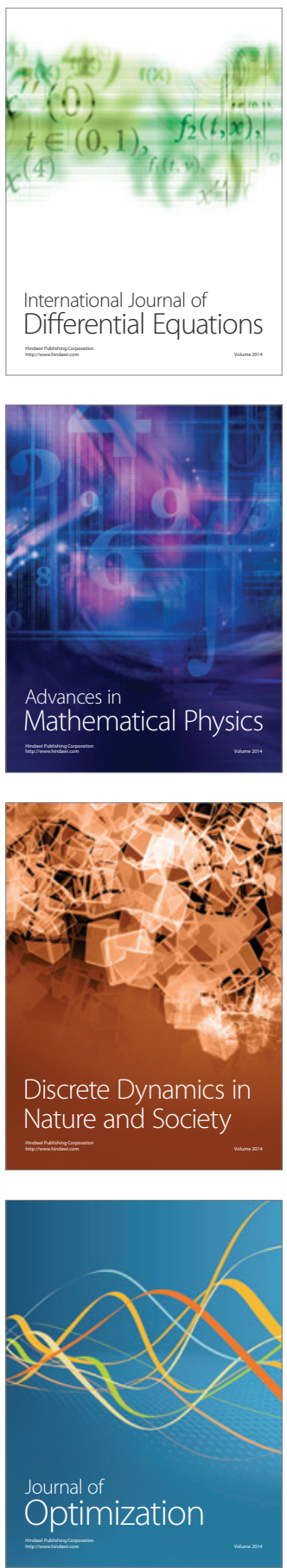